\title{
QCD analysis of non-singlet structure functions
}

\author{
A. Ghaffari-Turan ${ }^{1, a}$, A. Khorramian ${ }^{1, b}$, and H. Abdolmaleki ${ }^{1, c}$ \\ ${ }^{1}$ Faculty of Physics, Semnan University, 35131-19111, Semnan, Iran
}

\begin{abstract}
We present the results of the leading order (LO) and next-to-leading order (NLO) QCD analysis of experimental data in deep-inelastic scattering which relate to non-singlet structure functions. We use HERAFitter package to study the non-singlet $x F_{3}$ structure function. In this regards, we use the non-singlet structure function in $x$ space and we combine CCFR experimental data with NuTeV, CDHSW and CHORUS experimental data as well. Our results at LO and NLO are compatible with the available theoretical models.
\end{abstract}

\section{Introduction}

To obtain the nucleon structure functions in lepton-nucleon scattering or a physical cross section in nucleon-nucleon scattering, we need to have parton distribution functions (PDFs). The PDFs can not be extracted from the theory and in fact, they must be determined from the fits of the experimental data. These distributions are functions of $x$ and $Q^{2}$, and they are not only correlated by measurements from a wide variety of different processes and experiments, but also are correlated by theoretical constraints.

One of the most important structure functions in the neutrino-nucleon deep inelastic scattering, is the non-singlet structure function. However, the non-singlet structure function $x F_{3}$ is the main source to study the valence quark distributions $x u_{v}\left(x, Q^{2}\right)$ and $x d_{v}\left(x, Q^{2}\right)$ in QCD. The $Q^{2}$ dependence of these non-singlet distributions can obtain using the DGLAP evolution equations [1]. In this regard, the experimental data in the special range of deep inelastic scattering (DIS), is very important to determine the flavor of non-singlet parton distribution functions.

In recent years, several results of our QCD analysis about non-singlet structure functions in different cases are reported in Refs. [2-11]. In Ref. [2], we extracted valence quark distributions using QCD analysis of non-singlet parton distributions based on polynomials approach, such as Bernstein polynomials, and only CCFR data [12]. In the present paper, several data sets such as NuTeV [13] and CDHSW [14] and CHORUS [15] are included in the new analysis. So we can calculate the non-singlet structure function to determine valence quark distributions, using fits of the mentioned experimental data which are sensitive for determination of valence quark distributions.

Generally, the QCD analysis of parton distribution functions of DIS are accomplished at next-toleading order (NLO). Although the NLO approximation, as a standard approximation, is important

\footnotetext{
ae-mail: A.Ghafary@semnan.ac.ir

be-mail: Khorramiana@semnan.ac.ir,Khorramiana@theory.ipm.ac.ir

ce-mail: Abdolmaleki@semnan.ac.ir
} 
for the most processes in QCD, but next-to-next-to-leading order (NNLO) approximation is useful to have a higher precise [4]. In Ref. [16] non-singlet coefficient functions for charged-current DIS is reported in the third order as well.

The plan of the paper is as following. Formalism of the non-singlet structure function in QCD is given in Sec. 2. Our parametrization of parton densities are also presented in this section. Sec. 3 we summarize our results and discussions.

\section{Formalism}

In this section, we present the non-singlet structure function in $x$-space. The $x F_{3}$ non-singlet of structure function, represents the momentum distribution function of valence quarks.

In the LO approximation, the average of neutrino and anti-neutrino structure functions is related to non-singlet $x F_{3}(x)$ structure function and can be defined as [2]

$$
x F_{3}(x)=\frac{x F_{3}^{v N}+x F_{3}^{\bar{\nu} N}}{2},
$$

here $x F_{3}^{\nu N}$ and $x F_{3}^{\bar{\nu} N}$ is combination of PDFs as

$$
\begin{aligned}
& x F_{3}^{v N}=x u_{v}(x)+x d_{v}(x)+2 x s(x)-2 x c(x), \\
& x F_{3}^{\bar{v} N}=x u_{v}(x)+x d_{v}(x)-2 x s(x)+2 x c(x) .
\end{aligned}
$$

In above equations $u_{v}$ and $d_{v}$ are the valence quark distribution functions. So the average of the neutrino and anti-neutrino structure functions in above equations, is as following

$$
x F_{3}(x)=\frac{x F_{3}^{v N}+x F_{3}^{\bar{\nu} N}}{2}=x u_{v}(x)+x d_{v}(x),
$$

so it is clear that the $x F_{3}(x)$ structure function is only depend on the combination of valence quark distributions. In this analysis, and at the input scale of $Q_{0}^{2}=1.4 \mathrm{GeV}^{2}$ we choose the following valence quark distributions:

$$
\begin{gathered}
x u_{v}\left(x, Q_{0}^{2}\right)=N_{u_{v}} x^{a_{u_{v}}}(1-x)^{b_{u_{v}}}\left(1+c_{u_{v}} \sqrt{x}+d_{u_{v}} x\right), \\
x d_{v}\left(x, Q_{0}^{2}\right)=\frac{N_{d_{v}}}{N_{u_{v}}}(1-x)^{b_{d_{v}}} x u_{v}\left(x, Q_{0}^{2}\right)
\end{gathered}
$$

In the above equations, the term of $x^{a_{u_{v}}}$ controls the low- $x$ behavior parton distribution, and the term of $(1-x)^{b_{u_{v}}}$ is important in the large $x$ values. Other polynomial factors control the parton distribution for additional medium- $x$ values. Also the parameter $b_{d_{v}}$ is important to control the behavior of $x d_{v}$ for large $x$ value.

The above parametrization is similar to parametrization which is reported in Ref. [2]. The difference between QCD analysis in Ref. [2] and our new analysis is the different kinds of experimental data. In Ref. [2] we chose the CCFR experimental data and we used the QCD evolution of valance quarks distribution functions in $N$-Mellin space. In this analysis, we use the non-singlet structure function in $x$ space and also we combine CCFR with NuTeV, CDHSW and CHORUS experimental data as well. According to Ref. [17], the benefit of $x$-space approach is that we can choose any functional form at the input parton distribution in QCDnum package.

In above parametrization, the normalization constants $N_{u_{v}}$ and $N_{d_{v}}$ are fixed by: 


$$
\begin{aligned}
& \int_{0}^{1} u_{v} d x=2, \\
& \int_{0}^{1} d_{v} d x=1,
\end{aligned}
$$

so the normalization constants $N_{u_{v}}$ and $N_{d_{v}}$ are as following

$$
\begin{gathered}
N_{u_{v}}=\frac{2}{B\left(a_{u_{v}}, 1+b_{u_{v}}\right)+c_{u_{v}} B\left(1 / 2+a_{u_{v}}, 1+b_{u_{v}}\right)+d_{u_{v}} B\left(1+a_{u_{v}}, 1+b_{u_{v}}\right)}, \\
N_{d_{v}}=\frac{1}{B\left(a_{u_{v}}, 1+b_{u_{v}}+b_{d_{v}}\right)+c_{u_{v}} B\left(1 / 2+a_{u_{v}}, 1+b_{u_{v}}+b_{d_{v}}\right)+d_{u_{v}} B\left(1+a_{u_{v}}, 1+b_{u_{v}}+b_{d_{v}}\right)} .
\end{gathered}
$$

In above, $B(a, b)$ is the Euler Beta function.

The normalization constants $N_{u_{v}}$ and $N_{d_{v}}$ are very effective to determine unknown parameters in Eqs. $(5,6)$ via the QCD fitting procedure. We use also the evolution of strong coupling up to NLO which is given [17]:

$$
\frac{d a_{s}\left(\mu^{2}\right)}{\mathrm{d} \ln \mu^{2}}=-\sum_{i=0}^{1} \beta_{i} a_{s}^{i+2}(\mu),
$$

here $a_{s}=\alpha_{s} / 2 \pi$ and $\beta_{i}$ denote the expansion coefficients of the QCD $\beta$-function which are depend on the number of active quark flavor $n_{f}$, and in the $\overline{\mathrm{MS}}$ scheme they are given by $[18,19]$

$$
\begin{aligned}
& \beta_{0}=\frac{11}{2}-\frac{1}{3} n_{f}, \\
& \beta_{1}=\frac{51}{2}-\frac{19}{6} n_{f} .
\end{aligned}
$$

Note that, an analytical solution of Eq. (11) can be written at the leading order,

$$
\frac{1}{\alpha_{\mathrm{s}}\left(\mu^{2}\right)}=\frac{1}{\alpha_{\mathrm{s}}\left(\mu_{0}^{2}\right)}+\beta_{0} \ln \left(\frac{\mu^{2}}{\mu_{0}^{2}}\right) .
$$

By definition of $\Lambda_{Q C D}$, we can ignore the first term at the right-hand side of above equation, where at this scale the coupling constant could be infinite, i.e.,

$$
\frac{1}{\alpha_{\mathrm{s}}\left(\mu^{2}\right)}=\beta_{0} \ln \left(\frac{\mu^{2}}{\Lambda_{\mathrm{QCD}}^{2}}\right) .
$$

Since our QCD analysis is performed up to NLO, it is convenient to take $\alpha_{\mathrm{s}}\left(m_{\mathrm{Z}}^{2}\right)$ as a reference. The values of $\alpha_{\mathrm{s}}$ at any other scales are obtained using a numerical integration of Eq. (11) instead of approximate analytical solutions. The NNLO QCD Beta function is also reported in Ref. [20].

So the five unknown parameters can be extracted by the fitting procedure of the experimental data, with using HERAFitter package [21]. We add the mentioned experimental data which we need to study non-singlet structure function in HERAFitter package and we put our parametrization in this package as well. 


\section{Conclusion}

In this section, we describe our QCD fit results using the experimental data which we choose to study of non-singlet structure function. As we mentioned before, the experimental data which we used in this analysis at LO and NLO approximation are CCFR, NuTeV, CDHSW and CHORUS. In fact we include the experimental data which collected around 283 data points. During the fitting process, it was noted that functional form is unchanged from which used in our previous analysis [2]. In Fig. 1, the quality of our QCD fit to the data of non-singlet structure function are illustrated at LO and NLO. According to our fits, non-singlet $x F_{3}$ structure functions from experimental data are compared with the LO and NLO our fit results as a function of $x$ and at constant values of $Q^{2}$. In this case, we can survey the impact of NuTeV, CDHSW and CHORUS data when we combine them to CCFR experimental data as well.
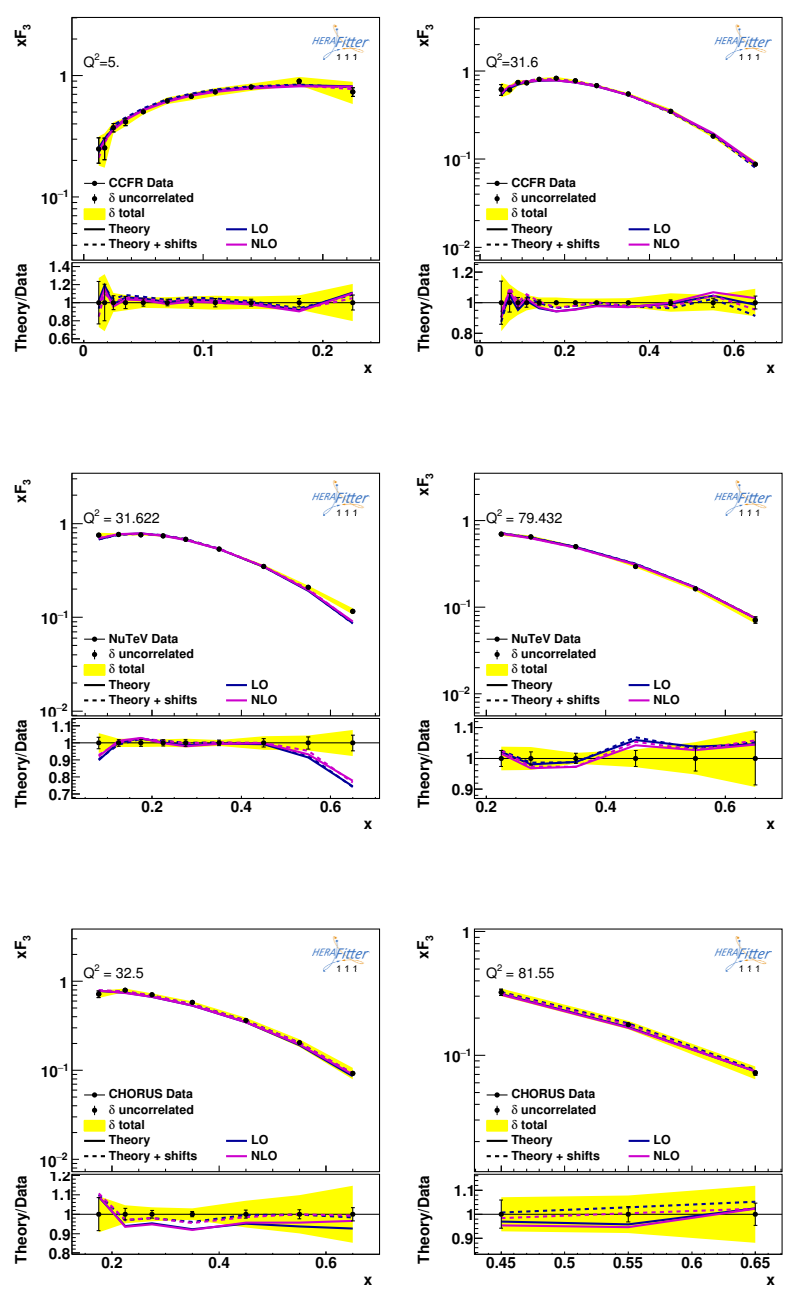

Figure 1. Comparison of $x F_{3}$ non-singlet structure function data with our QCD fit at $\mathrm{LO}$ and NLO approximation, as a function of $x$ and for fixed $Q^{2}$ values. 
The parameter values in Eqs. $(5,6)$ and their uncertainties for LO and NLO at the input scale of $Q_{0}^{2}$ are given in Table 1, with the parameters that are listed without uncertainty and fixed by constrains of Eqs. $(5,6)$. We fixed the strong coupling at the scale of $M_{Z}$ as a default value $\alpha_{s}\left(M_{Z}\right)=0.1185$, using the world average values which reported by the Particle Data Group [22].

Table 1. The fit results for parameter values at LO and NLO approximation and the input scale of $Q_{0}^{2}$ as defined in Eqs. $(5,6)$ using the experimental CCFR, NuTeV, CDHSW and CHORUS data.

\begin{tabular}{lll}
\hline \hline Parameter & LO & NLO \\
\hline \hline$N_{u_{v}}$ & 0.3236 & 2.5355 \\
\hline$a_{u_{v}}$ & $0.355 \pm 0.074$ & $0.635 \pm 0.096$ \\
\hline$b_{u_{v}}$ & $3.227 \pm 0.058$ & $3.180 \pm 0.060$ \\
\hline$c_{u_{v}}$ & $12.9 \pm 5.2$ & $-0.84 \pm 0.78$ \\
\hline$d_{u_{v}}$ & $3.4 \pm 1.9$ & $4.6 \pm 3.3$ \\
\hline$N_{d_{v}}$ & 0.2081 & 1.5163 \\
\hline$b_{d_{v}}$ & $1.65 \pm 0.43$ & $0.87 \pm 0.21$ \\
\hline \hline
\end{tabular}

According to Eqs. $(5,6)$ and using the extracted parameters from the fits of experimental data at the input scale of $Q_{0}^{2}$, which are shown in Table 1 , the $Q^{2}$ evolution for $x u_{v}$ and $x d_{v}$ valence quark distributions are possible. In this regards, the LO and NLO results for $x u_{v}$ and $x d_{v}$ valence quark distributions as a function of $x$ and for fixed different values of $Q^{2}=1,4,100,8317 \mathrm{GeV}^{2}$ are compared in Fig. (2) and Fig. (3) respectively.
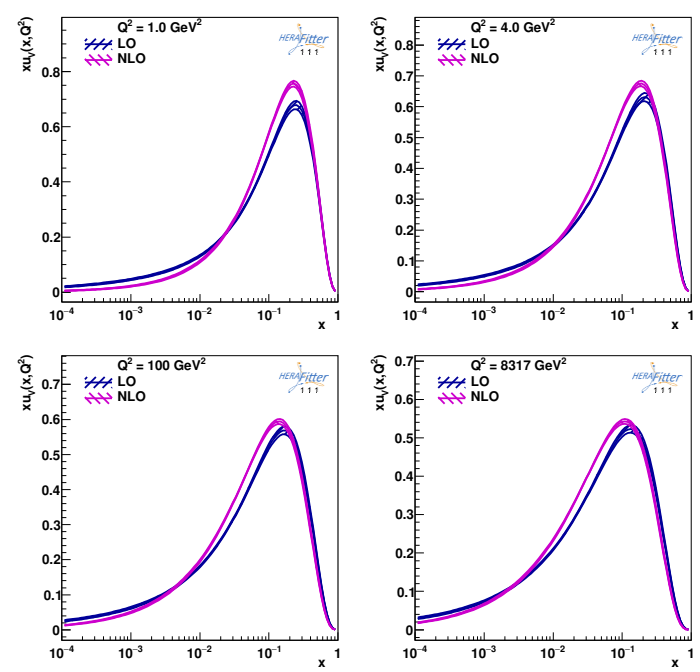

Figure 2. $x u_{v}$ valence quark distribution as a function of $x$ and for different values of $Q^{2}=1,4,100,8317 \mathrm{GeV}^{2}$. 

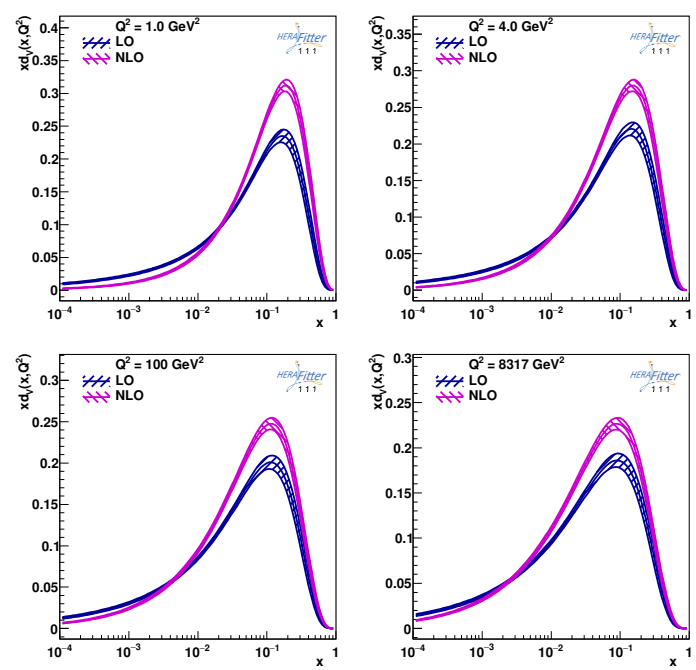

Figure 3. $x d_{v}$ valence quark distribution as a function of $x$ and for different values of $Q^{2}=1,4,100,8317 \mathrm{GeV}^{2}$.

\section{Acknowledgment}

We thank R. Placakyte for useful discussion on the xFitter framework. H. A. is grateful to the JINR (Joint Institute For Nuclear Research) and xFitter group for their hospitality and scholarship in xFitter meeting in Dubna.

\section{References}

[1] G. Altarelli and G. Parisi, Nucl. Phys. B 126, 298 (1977).

[2] A. N. Khorramian and S. Atashbar Tehrani, JHEP 0703, 051 (2007) [hep-ph/0610136].

[3] A. N. Khorramian and S. A. Tehrani, Phys. Rev. D 78, 074019 (2008) [arXiv:0805.3063 [hep$\mathrm{ph}]]$.

[4] A. N. Khorramian, H. Khanpour and S. A. Tehrani, Phys. Rev. D 81, 014013 (2010) [arXiv:0909.2665 [hep-ph]].

[5] S. Atashbar Tehrani and A. N. Khorramian, Nucl. Phys. Proc. Suppl. 186, 58 (2009).

[6] A. N. Khorramian and S. A. Tehrani, J. Phys. Conf. Ser. 110, 022022 (2008).

[7] A. N. Khorramian, S. A. Tehrani and M. Ghominejad, Acta Phys. Polon. B 38, 3551 (2007).

[8] A. N. Khorramian, S. A. Tehrani and A. Mirjalili, Nucl. Phys. Proc. Suppl. 164, 34 (2007).

[9] A. N. Khorramian and S. Atashbar Tehrani, eConf C 0605151, 0014 (2006) [hep-ph/0610172].

[10] A. N. Khorramian, H. Khanpour and S. Atashbar Tehrani, PoS EPS -HEP2009, 393 (2009).

[11] M. Soleymaninia and A. N. Khorramian, Int. J. Mod. Phys. A 26, 686 (2011).

[12] W. G. Seligman et al., Phys. Rev. Lett. 79, 1213 (1997) [hep-ex/9701017].

[13] D. Naples et al. [NuTeV Collaboration], [hep-ex/0307005].

[14] J. P. Berge et al., Z. Phys. C 49, 187 (1991).

[15] G. Onengut et al. [CHORUS Collaboration], Phys. Lett. B 632, 65 (2006).

[16] J. Davies, A. Vogt, S. Moch and J. A. M. Vermaseren, arXiv:1606.08907 [hep-ph]. 
[17] M. Botje, Comput. Phys. Commun. 182, 490 (2011) [arXiv:1005.1481 [hep-ph]].

[18] W. Furmanski and R. Petronzio, Z. Phys. C 11, 293 (1982).

[19] O. V. Tarasov, A. A. Vladimirov and A. Y. Zharkov, Phys. Lett. 93B, 429 (1980).

[20] S. A. Larin and J. A. M. Vermaseren, Phys. Lett. B 303, 334 (1993).

[21] S. Alekhin et al., Eur. Phys. J. C 75, no. 7, 304 (2015) [arXiv:1410.4412 [hep-ph]].

[22] K. A. Olive et al. [Particle Data Group Collaboration], Chin. Phys. C 38, 090001 (2014). 